\title{
HAND2-mediated proteolysis negatively regulates the function of estrogen receptor $\alpha$
}

\author{
TOMOHIKO FUKUDA*, AKIRA SHIRANE*, OSAMU WADA-HIRAIKE, KATSUTOSHI ODA, \\ MICHIHIRO TANIKAWA, AYAKO SAKUABASHI, MANA HIRANO, HOUJU FU, YOSHIHIRO MORITA, \\ YUICHIRO MIYAMOTO, KANAKO INABA, KEI KAWANA, YUTAKA OSUGA and TOMOYUKI FUJII
}

Department of Obstetrics and Gynecology, Graduate School of Medicine, The University of Tokyo, Tokyo 113-8655, Japan

Received October 2, 2014; Accepted June 11, 2015

DOI: $10.3892 / \mathrm{mmr} .2015 .4070$

\begin{abstract}
A previous study demonstrated that the progesterone-inducible HAND2 gene product is a basic helix-loop-helix transcription factor and prevents mitogenic effects of estrogen receptor $\alpha(E R \alpha)$ by inhibiting fibroblast growth factor signalling in mouse uteri. However, whether HAND2 directly affects the transcriptional activation function of ER $\alpha$ remains to be elucidated. In the present study, the physical interaction between HAND2 and ER $\alpha$ was investigating by performing an immunoprecipitation assay and an in vitro pull-down assay. The results demonstrated that HAND2 and ER $\alpha$ interacted in a ligand-independent manner. The in vitro pull-down assays revealed a direct interaction between HAND2 and the amino-terminus of ER $\alpha$, termed the activation function-1 domain. To determine the physiological significance of this interaction, the role of HAND2 as a cofactor of ER $\alpha$ was investigated, which revealed that HAND2 inhibited the ligand-dependent transcriptional activation function of ER $\alpha$. This result was further confirmed and the mRNA expression of vascular endothelial growth factor, an ER $\alpha$-downstream factor, was decreased by the overexpression of HAND2. This inhibition of ligand-dependent
\end{abstract}

Correspondence to: Dr Osamu Wada-Hiraike, Department of Obstetrics and Gynecology, Graduate School of Medicine, The University of Tokyo, 7-3-1 Hongo, Bunkyo-ku, Tokyo 113-8655, Japan E-mail: osamuwh-tky@umin.ac.jp

*Contributed equally

Abbreviations: AF, activation function; AhR, aryl hydrocarbon receptor; ARNT, aryl hydrocarbon receptor nuclear translocator; AZA, Aza-2'-deoxycytidine; bHLH, basic helix-loop-helix; $\mathrm{E}_{2}$, $17 \beta$-estradiol; ER, estrogen receptor; FGF, fibroblast growth factor; GAPDH, glyceraldehyde 3-phosphate dehydrogenase; MPA, medroxyprogesterone acetate; PPT, propylpyrazole triol; Ub, ubiquitin; UFD1L, ubiquitin fusion degradation 1 L; VEGF, vascular endothelial growth factor

Key words: endometrial cancer, HAND2, estrogen receptor $\alpha$, ubiquitination transcriptional activation function of ER $\alpha$ was possibly attributed to the proteasomic degradation of ER $\alpha$ by HAND2. These results indicate a novel anti-tumorigenic function of HAND2 in regulating ER $\alpha$-dependent gene expression. Considering that HAND2 is commonly hypermethylated and silenced in endometrial cancer, it is hypothesized that HAND2 may serve as a possible tumor suppressor, particularly in uterine tissue.

\section{Introduction}

Endometrial cancer is one of the most common types of gynecologic malignancy, increasing each year (1). Based on a pathological view, endometrial cancer can be divided into two subtypes (2). Type 1 endometrial cancer includes highly differentiated endometrioid adenocarcinoma, which is characterized by stepwise carcinogenesis through endometrial hyperplasia to endometrial cancer. Type 2 endometrial cancer, including poorly differentiated, serous and clear cell adenocarcinoma, is reported to occur alongside de novo mutation of TP53 (3). The principal cause of type 1 endometrial cancer is considered to be the prolonged exposure to estrogens without antagonistic effect of progesterone, and this pathophysiology is closely associated with first grade amenorrhea, polycystic ovary syndrome, obesity and hormonal supplementation therapy (3). Estrogen replacement therapy is utilized to control menopausal symptoms, however, it is known to increase the risk of developing endometrial cancer between 2- and 20-fold for females possessing uteri (4), and the increment of risk is well correlated with the duration of its use. In order to reduce the risk of endometrial cancer, it is recommended that postmenopausal females possessing uteri use progestin together with estrogens (4). A representative progestin, medroxyprogesterone acetate (MPA) is used for fertility-sparing treatment in type 1 endometrial cancer (5). The way in which MPA maintains quiescence of the endometrium remains to be elucidated, although MPA is known to possess detrimental effects on breast tissue, and MPA marginally increases the risk of developing breast cancer (6). Therefore, analysis of the physiological role of estrogens for the prevention of endometrial cancer is urgently required.

HAND1 and HAND2 constitute the HAND subclass of the basic helix-loop-helix (bHLH) family, and were independently identified during analyses to identify candidate E-box 
binding transcription factors in yeast two-hybrid screens (7). HAND2 is known as a critical regulator of morphogenesis in a variety of tissues, as HAND2 is expressed in the heart and neural crest-derived tissues, and is essential for the formation of the brachial arch, cardiovasculature and limbs (8). It has been reported that HAND2 interacts with GATA4, Nkx2.5, MEF2C, Phox2 and Mash1 (9-12). GATA4, Nkx2.5 and $\mathrm{MEF} 2 \mathrm{C}$ are associated with cardiogenesis, whereas Phox 2 and Mash1 are associated with the development of the autonomic nervous system (9-12). It has been demonstrated that HAND2 forms homo- or heterodimers with other bHLH proteins, and activates transcription by binding to the E-box elements $(13,14)$. However, the downstream factors of HAND2 and the associations between HAND2 and nuclear receptors remain to be fully elucidated. A previous study revealed that HAND2 is localized exclusively in the uteri of stromal tissue, and progesterone-induced expression of HAND2 in the murine uterine stroma suppresses the production of fibroblast growth factors (FGFs), which act as paracrine mediators of the mitogenic effects of estrogen on the uterine epithelium (15). Whether HAND2 affects the transcriptional activation function of $\mathrm{ER} \alpha$ as a transcriptional factor remains to be elucidated.

Considering previous data, demonstrating that the expression of HAND2 is impaired in endometrial cancer, compared with normal endometrium and endometrial hyperplasia, as determined using DNA methylation analysis (16), the present study aimed to investigate the interaction between HAND2 and $\mathrm{ER} \alpha$, and aimed to identify the physiological function of HAND2, particularly associated with endometrial cancer. The results of this investigation may prove to be useful in identifying novel molecular-targeted therapies for the treatment of endometrial cancer.

\section{Materials and methods}

Chemicals and antibodies. The MG132 proteasome inhibitor, $17 \beta$-estradiol $\left(\mathrm{E}_{2}\right)$ and anti-Flag M2 agarose were purchased from Sigma-Aldrich (St. Louis, MO, USA). The ER $\alpha$ selective ligand, propylpyrazole triol (PPT), was obtained from Tocris Bioscience (Ellisville, MO, USA). Mouse monoclonal antibodies used were anti-ER $\alpha$ (D-12; Santa Cruz Biotechnology, Inc., Santa Cruz, CA, USA), anti- $\beta$-Actin (cat no. sc-47778; Santa Cruz Biotechnology, Inc.) and anti-HA (cat. no. 12CA5; Roche Applied Science, Basel, Switzerland). Rabbit polyclonal antibodies included anti-ER $\alpha$ (MC-20; Santa Cruz Biotechnology, Inc.), anti-ER $\beta$ (H-150; Santa Cruz Biotechnology, Inc.), anti-DYKDDDDK Tag (cat. no. \#2368 Cell Signaling Technology, Inc., Danvers, MA, USA), and anti-HAND2 (cat. no. PAB4702; Abnova, Taipei, Taiwan).

Cell culture. The ER $\alpha$-positive MCF-7 (cat. no. HTB-22) and ER $\beta$ positive MDA-MB-231 (cat. no. HTB-26) human breast cancer cell lines, and the AN3CA human endometrial cancer cell line (cat. no. HTB-111) were purchased from American Type Culture Collection (Manassas, VA, USA). These cells were maintained in Dulbecco's modified Eagle's medium (Wako Pure Chemical Industries, Ltd., Osaka, Japan) supplemented with $10 \%$ fetal bovine serum (Sigma-Aldrich) at $37^{\circ} \mathrm{C}$ in a humidified $5 \% \mathrm{CO}_{2}$ incubator.
Expression vectors. Human ER $\alpha$ vectors and the ERE-tk-Luc and 17M8-AdMLP-Luc reporter constructs were used, as described previously $(17,18)$.

Immunoprecipitation, western blot analysis and ubiqutination assay. The formation of endogenous HAND2-ER $\alpha$ and HAND2-ER $\beta$ complexes were analysed by co-immunoprecipitation using specific antibodies raised against human $\mathrm{ER} \alpha$ and $\mathrm{ER} \beta$, followed by immunoblotting using anti-human HAND2 antibody, as described previously $(17,18)$.

For evaluating the HAND2-mediated degradation of ER $\alpha$, $2 \times 10^{5}$ AN3CA cells were transfected with $0.2 \mu \mathrm{g} / \mathrm{ml}$ pcDNA FLAG ER $\alpha$ and/or pcDNA Myc HAND2 in $6 \mathrm{~cm}$ dishes. The cells were treated with or without MG132 $\left(10^{-5} \mathrm{M}\right)$, and were harvested $24 \mathrm{~h}$ following the addition of MG132.

For evaluating the HAND2-mediated degradation of ER $\alpha, 0.2 \mu \mathrm{g} / \mathrm{ml}$ HA-tagged ubiquitin (HA-Ub), pcDNA FLAG ER $\alpha$ and pcDNA Myc HAND2 were transfected into $4 \times 10^{4} \mathrm{HEK} 293 \mathrm{~T}$ cells, in the presence or absence of $\mathrm{E}_{2}$ $\left(10^{-8} \mathrm{M}\right)$. The cell lysates were subjected to anti-Flag M2 agarose (1:100; Sigma-Aldrich) and the level of Ub-bound $\mathrm{ER} \alpha$ protein was evaluated using western blotting, as previously described $(17,18)$.

The antibodies used for western blotting were as follows: Anti-ER $\alpha$ (1:1,000; mouse monoclonal and rabbit polyclonal), anti- $\beta$-actin $(1: 10,000)$, anti-HA $(1: 1,000)$, anti-ER $\beta(1: 1,000)$, anti-DYKDDDDK Tag $(1: 1,000)$ and anti-HAND2 $(1: 1,000)$. These primary antibodies were incubated overnight at $4{ }^{\circ} \mathrm{C}$ and the results were visualized by ImageQuant ${ }^{\mathrm{TM}}$ LAS-3000 (GE Healthcare Life Sciences, Chalfont, UK).

In vitro glutathione $S$-transferase (GST)-pull down assay. The GST fusion proteins, GST-ER $\alpha$ activation function (AF)-1/AF-2, or GST alone were expressed in Escherichia coli (Takara Bio, Inc., Otsu, Japan) and bound to glutathione-sepharose 4B beads (GE Healthcare Life Sciences). The expression levels of GST-ER $\alpha$ AF-1 and AF-2 were confirmed using Coomassie Brilliant Blue (Thermo Fisher Scientific, Waltham, MA, USA) staining $(17,18)$. The immobilized GST-ER $\alpha$ AF-2 fusion proteins were preincubated for $30 \mathrm{~min}$ in GST binding buffer, containing $20 \mathrm{mM}$ Tris- $\mathrm{HCl}$ (pH 7.5), $200 \mathrm{mM} \mathrm{NaCl}$ and $1 \mathrm{mM}$ EDTA, with or without $\mathrm{E}_{2}\left(10^{-6} \mathrm{M}\right)$. The GST proteins were incubated at $4^{\circ} \mathrm{C}$ with the indicated $\left.{ }^{35} \mathrm{~S}\right]$ methionine-labeled proteins. After $1 \mathrm{~h}$ incubation, unbound proteins were removed by washing the beads in GST binding buffer containing $0.5 \%$ Nonidet P-40 (Wako Pure Chemical Industries, Ltd.) and protease inhibitor cocktail (Roche Applied Science). The specifically-bound proteins were eluted by boiling in SDS sample buffer and analysed using $10 \%$ SDS polyacrylamide gel electrophoresis and autoradiography (ImageQuant ${ }^{\mathrm{TM}}$ LAS-3000; GE Healthcare Life Sciences).

Luciferase reporter assay. For the luciferase assay, 4x10 ${ }^{4}$ HEK293T cells were transfected with $0.2 \mu \mathrm{g} / \mathrm{ml}$ pcDNA, pcDNA FLAG ER $\alpha$, pcDNA, pM ER $\alpha$ AF-2 and 0.2-0.6 $\mu \mathrm{g} / \mathrm{ml}$ pcDNA Myc HAND2 vectors using Effectene reagent (Qiagen, Hilden, Germany), according to the manufacturer's instructions. As an internal control to equalize the transfection efficiency, a phRL CMV-Luc vector (Promega 
Corporation, Madison, WI, USA) was also transfected in all the experiments. Individual transfections, each consisting of triplicate wells, were repeated at least three times $(17,18)$.

Reverse transcription-semi-quantitative polymerase chain reaction $(R T-q P C R)$. The AN3CA cells were transfected with either pcDNA3 (control; Invitrogen, Carlsbad, CA, USA) or pcDNA Myc HAND2. These cells were then treated with vehicle, $\mathrm{E}_{2}$ or PPT for $24 \mathrm{~h}$. Total RNA was extracted from the cells using an RNeasy Mini kit (Qiagen), and cDNA was synthesized using ReverTra Ace (Toyboyo, Co, Ltd., Tokyo, Japan). The expression of each mRNA was normalised for RNA loading in each sample using glyceraldehyde 3-phosphate dehydrogenase (GAPDH). The primers and conditions for the amplification of GAPDH were as described previously (19). The PCR primers for GAPDH VEGF and ER $\alpha$ were as follows: GAPDH, forward 5'-TGCACCACCAAC TGCTTAGC-3' and reverse 5'-GGCATGGACTGTGGTCAT GAG-3; VEGF, forward 5'-CCAGCAGAAAGAGGAAAG AGGTAG-3' and reverse 5'-CCCCAAAAGCAGGTCACT CAC-3; and ER $\alpha$, forward 5'-TGTGCAATGACTATGCTT CA-3' and reverse 5'-GCTCTTCCTCCTGTTTTTA-3'. Firstly, 250 ng cDNA, $0.1 \mu \mathrm{l}$ Ex Taq Polymerase (Takara Bio, Inc.) and $0.2 \mu \mathrm{M}$ primers were mixed. Thereafter each PCR regimen involved a 2 min initial denaturation step $\left(94^{\circ} \mathrm{C}\right)$, which was followed by $15-30$ cycles at $94^{\circ} \mathrm{C}$ for $30 \mathrm{sec}$, then at $55^{\circ} \mathrm{C}$ for $30 \mathrm{sec}$ and finally at $72^{\circ} \mathrm{C}$ for $30 \mathrm{sec}$ using a Thermal Cycler Gene Atlas instrument (ASTEC Co., Ltd., Kasuya, Japan).

Statistical analysis. Data are presented as the mean \pm standard error of the mean from at least three independent determinations. Multiple comparisons between more than two groups were analysed using one-way analysis of variance and post-hoc tests using GraphPad Prism version 6.0 with the Bonferoni/Dunn post hoc tests (GraphPad Software, San Diego, CA, USA). $\mathrm{P}<0.05$ were considered to indicate a statistically significant difference.

\section{Results}

$H A N D 2$ directly binds to the AF-1 region of $E R \alpha$ in a ligand-independent manner. To assess the hypothesis that ER $\alpha$ interacts with HAND2 protein, the present study performed immunoprecipitation assays using antibodies raised against ER $\alpha$. The immunoblotting revealed the existence of HAND2 protein in the cell lysates of the ER $\alpha$-proficient MCF-7 cells (Fig. 1A). The present study also performed immunoprecipitation assays using antibodies raised against $\operatorname{ER} \beta$, and the immunoblotting revealed the existence of HAND2 protein in the cell lysates of the ER $\beta$-proficient MDA-MB-231 cell (Fig. 1B), which indicated that HAND2 was physically associated with ER $\alpha$ and ER $\beta$. Subsequently, the present study examined the function of HAND2 in association with ER $\alpha$ to determine the physiological function of HAND2 in endometrial cancer.

The results, described above, were further confirmed using in vitro pull-down assays to demonstrate the functional importance of the HAND2-ER $\alpha$ interaction. To map the region of ER $\alpha$ that interacts with HAND2 as a transcription factor, GST-fused ER $\alpha$ activation function (AF)-1 or
A
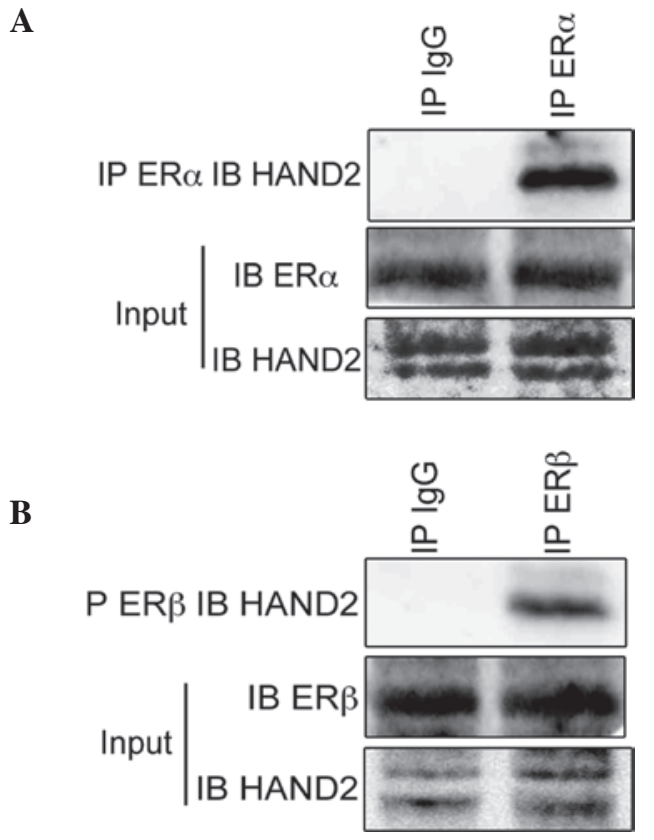

Figure 1. HAND2 forms an endogenous complex with ER $\alpha / \beta$. (A) Formation of the HAND2-ER $\alpha$ complex in MCF-7 cells was analysed by IP with the antibodies against $\mathrm{ER} \alpha$ or preimmune $\mathrm{IgG}$, followed by IB using the anti-HAND2 antibody. (B) Formation of the HAND2 and ER $\beta$ complex in MDA-MB-231 cells, analysed by IP with the antibodies to ER $\beta$ or preimmune $\mathrm{IgG}$, followed by IB using the anti-HAND2 antibody. Input denotes the whole cell lysate used for IP. IP, co-immunoprecipitation; IB, immunoblotting; $\mathrm{ER} \alpha$, estrogen receptor $\alpha$.

AF-2 (Fig. 2A) and $\left.{ }^{35} \mathrm{~S}\right]$ methionine-labelled HAND2 were incubated and their interactions were assessed. As shown in Fig. 2B, the GST-fused ER $\alpha$ AF-1 protein possessed the ability to retain HAND2 on the column. The GST ER $\alpha$ AF-2 column exhibited weak interaction with HAND2, compared with the GST ER $\alpha$ AF-1 column and the interaction between HAND2, and GST ER $\alpha$ AF-2 was unchanged, regardless of the presence of $\mathrm{E}_{2}$ (Fig. 2C). These data indicated that HAND2 interacted directly with ER $\alpha$ AF-1 in a ligand-independent manner.

HAND2 represses the transcriptional activation function of ER $\alpha$. To examine the cofactor activity of HAND2 in the transcriptional activation function of ER $\alpha$, the present study performed transient transfection assays using a luciferase reporter plasmid, driven by a thymidine kinase promoter containing three tandem repeats of the canonical estrogen responsive element (AGGTCAnnnTGACCT). Although ER $\alpha$ exhibited a ligand-dependent transactivation function in the HEK293T cells (Fig. 3A, lane 4), the transient expression of HAND2 led to a significant decrease in luciferase activity of $\mathrm{ER} \alpha$, and this downregulation increased as the quantity of HAND2 expression vector increased (Fig. 3A, lanes 5-7).

The present study subsequently aimed to determine the role of HAND2 in the transactivation function of GAL4-fused $\mathrm{ER} \alpha \mathrm{AF}-2$. For this purpose, transient transfection assays were performed in HEK293T cells using a 17M8-AdMLP-luc luciferase reporter plasmid (20). The ligand-induced transactivation function of ER $\alpha$ AF-2 (Fig. 3B, lane 4) was not significantly affected by the exogenous expression of HAND2 (Fig. 3B, lane 6). 
A

$E R \propto(1-595$ a.a.)

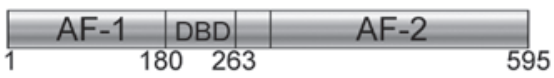

HAND2 (1-217 a.a.)

Transcription activation domain

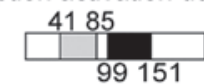

basic Helix-Loop-Helix

B

C

input ${ }^{35} \mathrm{~S}$-HAND2

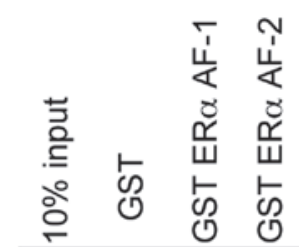

input ${ }^{35}$ S-HAND2

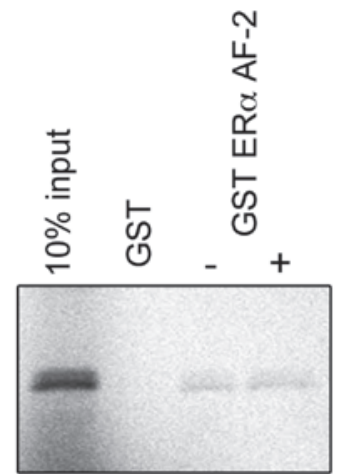

Figure 2. HAND2 physically associates with ER $\alpha / \beta$. (A) Schematic domain structures of ER $\alpha$ and HAND2. (B) Mapping of the HAND2-interaction region of ER $\alpha$ using GST-ER $\alpha$ AF-1, GST-ER $\alpha$ AF-2 and HAND2. (C) Analysis of the ligand-dependent association between HAND2 and the AF-2 region of ER $\alpha$. Bacterially expressed GST fusion proteins, immobilized on beads, were used for in vitro pull-down assays. Full-length HAND2 (amino acids 1-217) was translated in the presence of $\left[{ }^{35} \mathrm{~S}\right]$ methionine using a TNT-Coupled In Vitro Translation system. Labelled HAND2 was incubated with GST-ER $\alpha$ AF-1 or AF-2 with (+) or without (-) $10^{-6} \mathrm{M} \mathrm{E}_{2}$. The fragments of GST-ER $\alpha$ AF-1 and AF-2 were detected and the mixture was washed and subjected to SDS-polyacrylamide gel electrophoresis. Polyacrylamide gels were stained briefly with Coomassie Brilliant Blue to verify the loading of equal quantities of fusion proteins prior to drying and autoradiography. Input denotes in vitro translated protein. $\mathrm{ER} \alpha$, estrogen receptor $\alpha$; $\mathrm{AF}$, activation function; DBD, DNA binding domain; GST, glutathione S-transferase.

To evaluate the effect of HAND2 on endogenous gene expression, the mRNA expression of VEGF was examined in AN3CA endometrial cancer cells, as expression of the VEGF gene is driven by estrogen (21). As expected, treatment of the AN3CA cells with $\mathrm{E}_{2}$ led to a significant increase in the mRNA expression of VEGF, and overexpression of HAND2 suppressed the mRNA expression of VEGF, in the presence or absence of ER $\alpha$ agonists. However, suppression was most prominent in the presence of PPT, an ER $\alpha$ specific agonist (Fig. 3C). Therefore, these results indicated that HAND2 suppressed the transcriptional activation function of ER $\alpha$ via its AF-1 domain, and the suppression was specific for ER $\alpha$.
$\mathbf{A}$

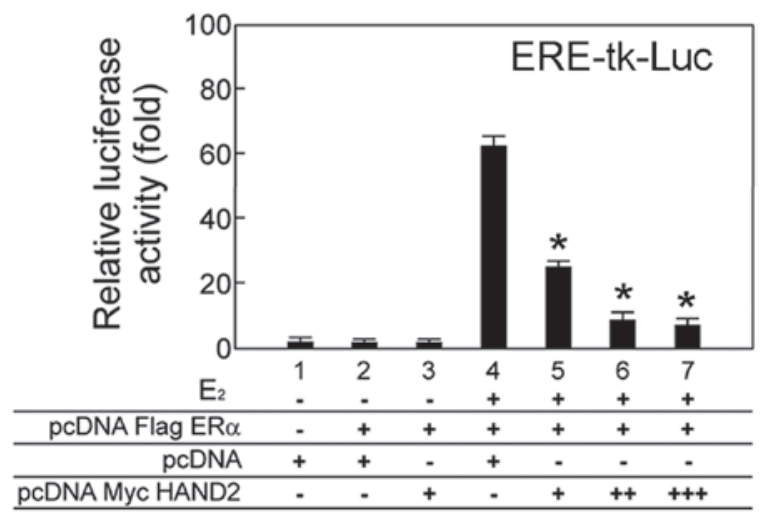

B
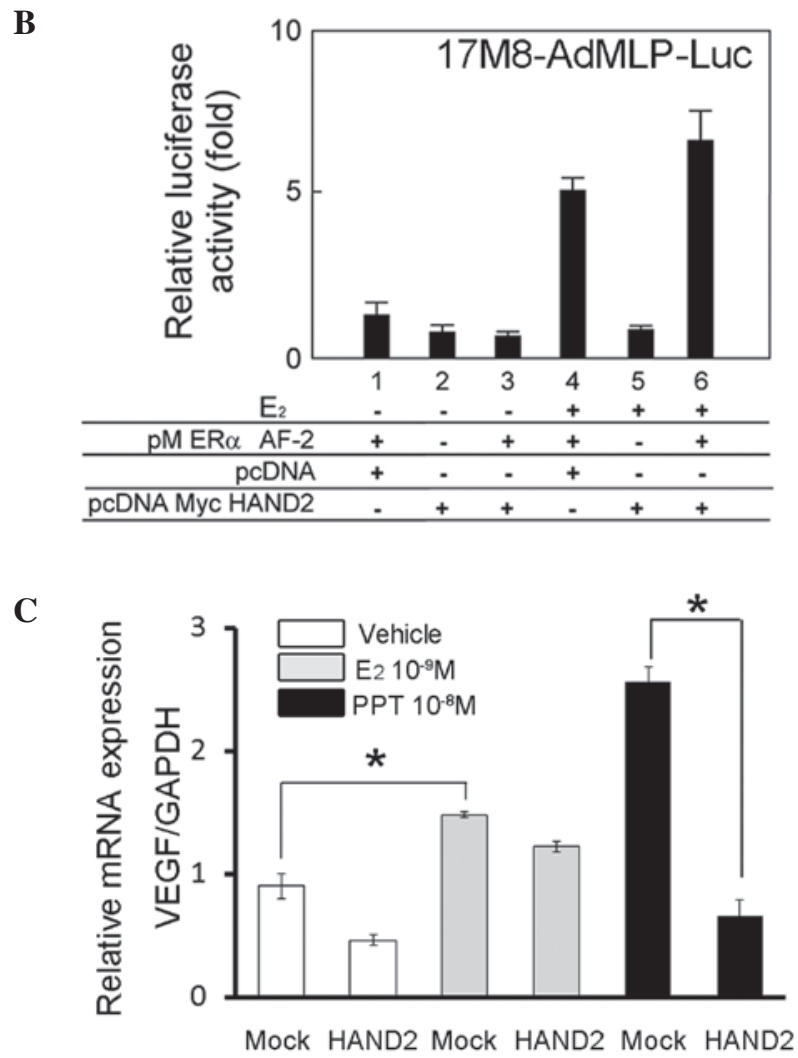

Figure 3. HAND2 attenuates the ligand-independent transcriptional activation function of ER $\alpha$. (A) Transient transfection assays were performed to examine the activity of HAND2 in the transcriptional activation function of ER $\alpha$. Indicated plasmids were cotransfected into the HEK293T cells in the presence or absence of $\mathrm{E}_{2}\left(10^{-8} \mathrm{M}\right)$. The cells were harvested $24 \mathrm{~h}$ after transfection with the expression vectors and reporter constructs (ERE-tk-Luc), and the transfected whole cell lysates were assayed for luciferase activity, produced from the reporter plasmid. HAND2 exhibited specific repression of the ligand-dependent transactivation function of ER $\alpha$ in the HEK293T cells, in a dose-dependent manner. Individual transfections, each consisting of triplicate wells, were repeated at least three times. ${ }^{~} \mathrm{P}<0.05$ vs. lane 4. (B) Transient transfection assays were performed to examine the activity of HAND2 in the transcriptional activation function of ER $\alpha$ AF-2. The cells were harvested $24 \mathrm{~h}$ after transfection with the expression vectors and reporter constructs (17M8-AdMLP-Luc), and the transfected whole cell lysates were assayed for luciferase activity, produced from the reporter plasmid. HAND2 exhibited no significant effect on the ligand-dependent transactivation function of ER $\alpha$ AF-2 in the HEK293T cells. Individual transfections, each consisting of triplicate wells, were repeated at least three times. (C) mRNA expression of VEGF was analysed as the representative downstream gene of $\mathrm{ER} \alpha$. The exogenous expression of HAND2 in the AN3CA cells decreased the mRNA level of VEGF, particuarly in the presence of PPT. mRNA levels were normalized by GAPDH. "P $<0.05$. Mock denotes transfection of empty (pcDNA) vector, and vehicle denotes solvent. ER $\alpha$, estrogen receptor $\alpha$; AF, activation function; PPT, propylpyrazole triol; VEGF, vascular endothelial growth factor; GAPDH, glyceraldehyde 3-phosphate dehydrogenase. 
A

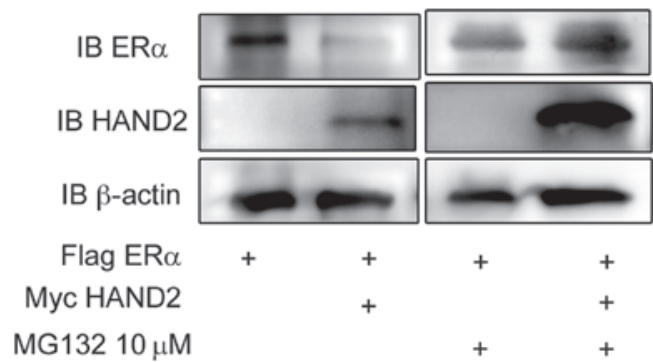

B

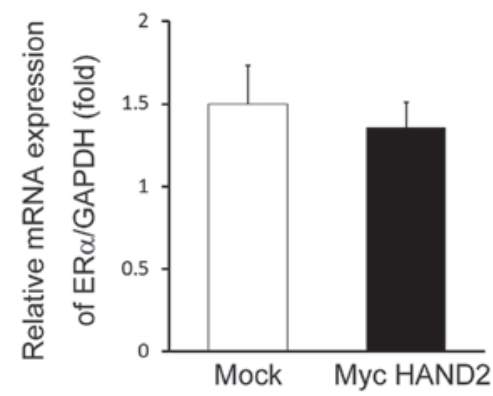

C

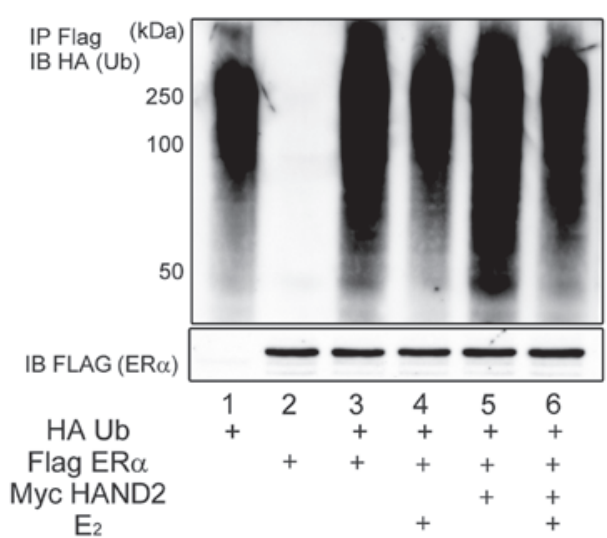

Figure 4. HAND2 decreases the expression of ER $\alpha$ by proteasomal degradation. (A) AN3CA cells were transfected with the expression vector of HAND2, in the absence or presence of MG132 $\left(10^{-5} \mathrm{M}\right)$ following transient transfection. The cells were harvested $48 \mathrm{~h}$ after transfection, and the whole cell lysates were subsequently extracted. The protein levels were analysed using western blotting with the indicated antibodies. The protein expression of ER $\alpha$ was significantly attenuated by the forced expression of HAND2, while the attenuation of expression of ER $\alpha$ expression by HAND2 was reversed in the presence of MG132. (B) AN3CA cells were transfected with the expression vector of HAND2 in the absence of $\mathrm{E}_{2}$, and the cells were harvested $48 \mathrm{~h}$ after transfection, followed by mRNA extraction. The forced expression of HAND2 had no significant effect on the mRNA expression of ER $\alpha$. (C) ER $\alpha$ was degraded via the ubiquitin-proteasome pathway. The HEK293T cells were cultured with the indicated vectors in the presence or absence of $\mathrm{E}_{2}\left(10^{-8} \mathrm{M}\right)$. Flag-tagged $\mathrm{ER} \alpha$ was immunoprecipitated (IP) using anti-Flag M2 antibody. The ubiquitination status of ER $\alpha$ was analysed using anti-HA antibody. The lower panel denotes the quantity of immunoprecipitated ER $\alpha$ detected by western blotting on probing with anti-Flag antibody. Mock denotes transfection of empty (pcDNA) vector. ER $\alpha$, estrogen receptor $\alpha$; IB, immunoblotting; HA-Ub, HA-tagged ubiquitin; GAPDH, glyceraldehyde 3-phosphate dehydrogenase.

Stimulation of proteasomal degradation by HAND2 decreases the expression level of ER $\alpha$. To elucidate the mechanism underlying the HAND2-induced decrease in the ligand-dependent transcriptional activation function of $E R \alpha$, the present study investigated the possibility of post-transcriptional modification of ER $\alpha$ by HAND2. The protein expression of ER $\alpha$ was significantly reduced by exog- enous expression of HAND2 in the AN3CA cells (Fig. 4A), although the mRNA level of ER $\alpha$ was unaffected by forced expression of HAND2 (Fig. 4B). In addition, the decreased protein expression of $\mathrm{ER} \alpha$ was reversed by the addition of the MG132 proteasome inhibitor (Fig. 4A). Thus, it was hypothesized that HAND2 protein may stimulate degradation of the $\mathrm{ER} \alpha$ protein, and this degradation of ER $\alpha$ protein results in the downregulation of transcriptional activation of ER $\alpha$. To confirm this hypothesis, HA-Ub and Flag-tagged ER $\alpha$ were transfected into the HEK293T cells, and the protein level of $\mathrm{ER} \alpha$ and ubiqutination status of ER $\alpha$ were determined using western blotting with anti-Flag M2 agarose and anti-HA antibody. The ubiquitination assays demonstrated the polyubiquitinated status of ER $\alpha$ (Fig. 4C), indicating that ER $\alpha$ was degraded via the ubiquitin-proteasome pathway, irrespective of the presence or absence of $E_{2}$.

\section{Discussion}

Although it has been reported that HAND2 belongs to the bHLH transcription factor, which binds to the E-box domain, its transcriptional function on ER $\alpha$ remained to be fully elucidated. The present study demonstrated that HAND2 functioned as a modifier of $E R \alpha$. Representative transcription factors that contain the bHLH domain include hypoxia-inducible factor (HIF), Myc, aryl hydrocarbon receptor (AhR), aryl hydrocarbon receptor nuclear translocator (ARNT) and TWIST1/2 (22), and the characterization of the bHLH family as a factor that affects the nuclear receptor superfamily has been investigated substantially. Originally, the interaction between AhR and ER $\alpha$ was identified through the investigation of 2,3,7,8-tetrachlorodibenzo-[p]-dioxin (TCDD) (23), and AhR was identified as a ligand-dependent ubiquitin E3 ligase $(24,25)$. AhR also belongs to the nuclear receptor superfamily, and the AhR/ARNT heterodimer inhibits ER $\alpha$ activity by binding to the AF-1 lesion of ER $\alpha$ (26), similar to HAND2 in the present study (Fig. 2B). In the present study, exogenous expression of HAND2 repressed the transcriptional activity of ER $\alpha$ (Fig. 3A and B). This mechanism is similar to that of AhR. Therefore, it is not surprising that HAND2 repressed the ligand-dependent transcriptional activation function of ER $\alpha$.

It has been accepted that the function of ER $\alpha$ is regulated at transcriptional and post-transcriptional levels. The latter includes phosphorylation, acetylation, sumoylation, methylation, palmitoylation, modulation by microRNA and ubiquitination (27). For recognition by ubiquitin ligases, a substrate protein requires phosphorylation or methylation. The sequential administration of ubiquitin activating enzyme (E1), ubiquitin conjugating enzyme (E2) and ubiquitin ligase (E3) is followed by $26 \mathrm{~S}$ proteasomal degradation (27). Several ubiquitin proteasome pathway components, including E6AP, MDM2, BRCA1 and $\mathrm{SCF}^{\mathrm{SKP} 2}$ are considered to be ER $\alpha$ cofactors (28). Among these, BRCA1, a breast and ovarian cancer-susceptible gene product, is known to form a complex with BARD1, and this complex decreases the ligand-dependent transcriptional activation function of ER $\alpha$ (29). The observation that HAND2 functioned as a co-repressor of ER $\alpha$ in the present study resembled the function of BRCA1, and this inhibition leads to the continuous suppression of transcriptional activity of ER $\alpha$, regardless of the presence of ligands. 
Although the expression of HAND2 is regulated by progesterone (15), a previous report suggested that increased calcineurin/Nfat signalling and decreased expression of miR-25 integrated to re-express HAND2 (30), and others have reported that the expression of HAND2 is silenced by methylation of the promoter lesion of HAND2 (16). The present study investigated whether the expression of HAND2 was manipulated by a DNA de-methylation chemical using 5-Aza-2'-deoxycytidine (AZA). However, the protein level of HAND2 remained unchanged following treatment of the AN3CA cells with AZA (data not shown). Therefore, another possible mechanism requires consideration to fully elucidate how the expression of HAND2 may be modulated. Although it has been demonstrated that the expression of HAND2 correlates with the ubiquitin fusion degradation 1L (UFD1L) ubiquitin-conjugating protein (31), the effects of HAND2 on the expression of UFD1 L were not examined in the present study. Clarification of the mechanism underlying how HAND2 recruits ubiquitin-proteasome machinery is required, as HAND2 itself is not an ubiquitin-conjugating enzyme.

The findings of the present study suggested that HAND2 may contribute to the suppression of tumorigenesis, as ER $\alpha$ generally contributes to tumorigenic function by stimulating cellular proliferation (32) and $\mathrm{E}_{2}$ may have a principal role in homeostasis of the uterine endometria, as continuous and unopposed stimulation of the uterine epithelia by $E_{2}$ results in the increased frequency of endometrial cancer (33). Consistent with this hypothesis, the expression of HAND2 was attenuated in the epithelia of endometrial cancer, compared with those in the normal endometrium and in endometrial hyperplasia (16), and the expression of VEGF was abrogated by concomitant overexpression of HAND2 particularly in the presence of the ER $\alpha$-specific ligand, PPT (Fig. 3C). It was suggested that HAND2 is involved in maintaining the quiescence of uterine endometria, however, determining the detailed mechanism underlying the effects on ER $\alpha$ by HAND2 requires further investigation. The present study also revealed the interaction between HAND2 and ER $\beta$. VEGF is a downstream factor of $\mathrm{ER} \beta$, as well as $\mathrm{ER} \alpha$, and $\mathrm{E}_{2}$ stimulates $\mathrm{ER} \alpha$ and $\mathrm{ER} \beta$ (34), however, the role of ER $\beta$ in endometrial cancer remains to be fully elucidated. The present study suggested that HAND2, a transcription factor for morphogenesis, may have a function in suppressing estrogen-dependent cancer. Breast cancer is known to be an estrogen-associated cancer, and the role of HAND2 in breast cancer remains to be elucidated. HAND2 may provide an important molecular target for these hormone-dependent types of cancer, and identification of the regulating mechanism of HAND2 may improve the control of these types of cancer.

In conclusion, the present study demonstrated the role of HAND2 as a negative transcriptional regulator of ER $\alpha$. HAND2 was involved in the inactivation of ER $\alpha$ by associating with the amino-terminus of ER $\alpha$, and exerted degradation of ER $\alpha$ by stimulating the ubiquitin-proteasome pathway. Therefore, in addition to inhibition of FGF signalling in the uterine tissue, HAND2 directly affected the mitogenic effects of ER $\alpha$, and these results suggested that inactivation of HAND2 may be detrimental in the regulation of cellular proliferation. Consistent with this hypothesis, hypermethylation and silencing of HAND2 is commonly found in endometrial cancer (16). Taken together, it can be hypothesized that HAND2 may serve as a possible tumor suppressor, particularly in uterine tissue. However, further investigations are required to confirm the physiological implications of HAND2 in the uterus.

\section{Acknowledgements}

The authors would like to thank Professor Murakami (Tokyo University of Science, Tokyo, Japan) for providing the HAND2 expression vector (pcDNA3.1 Myc-His B HAND2). This study was supported by a grant from the Grant-in-Aid for Scientific Research from the Ministry of Education, Science and Culture (grant no. 24592505).

\section{References}

1. SGO Clinical Practice Endometrial Cancer Working Group, Burke WM, Orr J, Leitao M, Salom E, Gehrig P, Olawaiye AB, Brewer M, Boruta D, Villella J, et al: Endometrial cancer: A review and current management strategies: Part I. Gynecol Oncol 134: 385-392, 2014.

2. Bokhman JV: Two pathogenetic types of endometrial carcinoma. Gynecol Oncol 15: 10-17, 1983.

3. Hecht JL and Mutter GL: Molecular and pathologic aspects of endometrial carcinogenesis. J Clin Oncol 24: 4783-4791, 2006.

4. Henderson BE, Casagrande JT, Pike MC, Mack T, Rosario I and Duke A: The epidemiology of endometrial cancer in young women. Br J Cancer 47: 749-756, 1983.

5. Koskas M, Uzan J, Luton D, Rouzier R and Daraï E: Prognostic factors of oncologic and reproductive outcomes in fertility-sparing management of endometrial atypical hyperplasia and adenocarcinoma: Systematic review and meta-analysis. Fertil Steril 101: 785-794, 2014.

6. Manson JE, Chlebowski RT, Stefanick ML, Aragaki AK, Rossouw JE, Prentice RL, Anderson G, Howard BV, Thomson CA, LaCroix AZ, et al: Menopausal hormone therapy and health outcomes during the intervention and extended poststopping phases of the Women's Health Initiative randomized trials. JAMA 310: 1353-1368, 2013.

7. Hollenberg SM, Sternglanz R, Cheng PF and Weintraub H: Identification of a new family of tissue-specific basic helix-loop-helix proteins with a two-hybrid system. Mol Cell Biol 15: 3813-3822, 1995.

8. Firulli AB: A HANDful of questions: The molecular biology of the heart and neural crest derivatives (HAND)-subclass of basic helix-loop-helix transcription factors. Gene 312: 27-40, 2003

9. Dai YS, Cserjesi P, Markham BE and Molkentin JD: The transcription factors GATA4 and dHAND physically interact to synergistically activate cardiac gene expression through a p300-dependent mechanism. J Biol Chem 277: 24390-24398, 2002.

10. Yamagishi H, Yamagishi C, Nakagawa O, Harvey RP, Olson EN and Srivastava D: The combinatorial activities of Nkx2.5 and dHAND are essential for cardiac ventricle formation. Dev Biol 239: 190-203, 2001.

11. Rychlik JL, Gerbasi V and Lewis EJ: The interaction between dHAND and Arix at the dopamine beta-hydroxylase promoter region is independent of direct dHAND binding to DNA. J Biol Chem 278: 49652-49660, 2003.

12. Morikawa Y, Dai YS, Hao J, Bonin C, Hwang S and Cserjesi P: The basic helix-loop-helix factor Hand 2 regulates autonomic nervous system development. Dev Dyn 234: 613-621, 2005.

13. Firulli BA, Hadzic DB, McDaid JR and Firulli AB: The basic helix-loop-helix transcription factors dHAND and eHAND exhibit dimerization characteristics that suggest complex regulation of function. J Biol Chem 275: 33567-33573, 2000.

14. Dai YS and Cserjesi P: The basic helix-loop-helix factor, HAND2, functions as a transcriptional activator by binding to E-boxes as a heterodimer. J Biol Chem 277: 12604-12612, 2002.

15. Li Q, Kannan A, DeMayo FJ, Lydon JP, Cooke PS, Yamagishi H, Srivastava D, Bagchi MK, Bagchi IC, et al: The antiproliferative action of progesterone in uterine epithelium is mediated by Hand2. Science 331: 912-916, 2011.

16. Jones A, Teschendorff AE, Li Q, Hayward JD, Kannan A, Mould T, West J, Zikan M, Cibula D, Fiegl H, et al: Role of DNA methylation and epigenetic silencing of HAND2 in endometrial cancer development. PLoS Med 10: e1001551, 2013. 
17. Wada-Hiraike O, Yano T, Nei T, Matsumoto Y, Nagasaka K, Takizawa S, Oishi H, Arimoto T, Nakagawa S, Yasugi T, et al: The DNA mismatch repair gene hMSH2 is a potent coactivator of oestrogen receptor alpha. Br J Cancer 92: 2286-2291, 2005.

18. Koyama S, Wada-Hiraike O, NakagawaS, Tanikawa M, Hiraike H, Miyamoto Y, Sone K, Oda K, Fukuhara H, Nakagawa K, et al: Repression of estrogen receptor beta function by putative tumor suppressor DBC1. Biochem Biophys Res Commun 392: 357-362, 2010.

19. Fu H, Wada-Hiraike O, Hirano M, Kawamura Y, Sakurabashi A Shirane A, Morita Y, Isono W, Oishi H, Koga K, et al: SIRT3 positively regulates the expression of folliculogenesis- and luteinization-related genes and progesterone secretion by manipulating oxidative stress in human luteinized granulosa cells. Endocrinology 155: 3079-3087, 2014.

20. Hiraike H, Wada-Hiraike O, Nakagawa S, Koyama S, Miyamoto Y, Sone K, Tanikawa M, Tsuruga T, Nagasaka K, Matsumoto Y, et al: Identification of DBC1 as a transcriptional repressor for BRCA1. Br J Cancer 102: 1061-1067, 2010

21. Cullinan-Bove K and Koos RD: Vascular endothelial growth factor/vascular permeability factor expression in the rat uterus: Rapid stimulation by estrogen correlates with estrogen-induced increases in uterine capillary permeability and growth. Endocrinology 133: 829-837, 1993.

22. Bersten DC, Sullivan AE, Peet DJ and Whitelaw ML: bHLH-PAS proteins in cancer. Nat Rev Cancer 13: 827-841, 2013.

23. White TE and Gasiewicz TA: The human estrogen receptor structural gene contains a DNA sequence that binds activated mouse and human Ah receptors: A possible mechanism of estrogen receptor regulation by 2,3,7,8-tetrachlorodibenzo-p-dioxin. Biochem Biophys Res Commun 193: 956-962, 1993.

24. Ohtake F, Baba A, Takada I, Okada M, Iwasaki K, Miki H, Takahashi S, Kouzmenko A, Nohara K, Chiba T, et al: Dioxin receptor is a ligand-dependent E3 ubiquitin ligase. Nature 446: $562-566,2007$

25. Swedenborg E and Pongratz I: AhR and ARNT modulate ER signaling. Toxicology 268: 132-138, 2010.
26. Ohtake F, Takeyama K, Matsumoto $\mathrm{T}$, Kitagawa $\mathrm{H}$, Yamamoto Y, Nohara K, Tohyama C, Krust A, Mimura J, Chambon P, et al: Modulation of oestrogen receptor signalling by association with the activated dioxin receptor. Nature 423 545-550, 2003.

27. Welboren WJ, Sweep FC, Span PN and Stunnenberg HG: Genomic actions of estrogen receptor alpha: What are the targets and how are they regulated? Endocr Relat Cancer 16: 1073-1089, 2009.

28. Zhou W and Slingerland JM: Links between oestrogen receptor activation and proteolysis: Relevance to hormone-regulated cancer therapy. Nat Rev Cancer 14: 26-38, 2014.

29. Fan S, Wang J, Yuan R, Ma Y, Meng Q, Erdos MR, Pestell RG, Yuan F, Auborn KJ, Goldberg ID, et al: BRCA1 inhibition of estrogen receptor signaling in transfected cells. Science 284: 1354-1356, 1999.

30. Dirkx E, Gladka MM, Philippen LE, Armand AS, Kinet V, Leptidis S, El Azzouzi H, Salic K, Bourajjaj M, da Silva GJ, et al: Nfat and miR-25 cooperate to reactivate the transcription factor Hand2 in heart failure. Nat Cell Biol 15: 1282-1293, 2013.

31. Yamagishi H, Garg V, Matsuoka R, Thomas T and Srivastava D: A molecular pathway revealing a genetic basis for human cardiac and craniofacial defects. Science 283: 1158-1161, 1999

32. Bondesson M, Hao R, Lin CY, Williams C and Gustafsson JA: Estrogen receptor signaling during vertebrate development. Biochim Biophys Acta 1849: 142-151, 2014.

33. Grady D, Rubin SM, Petitti DB, Fox CS, Black D, Ettinger B, Ernster VL and Cummings SR: Hormone therapy to prevent disease and prolong life in postmenopausal women. Ann Intern Med 117: 1016-1037, 1992.

34. Mueller MD, Vigne JL, Minchenko A, Lebovic DI, Leitman DC and Taylor RN: Regulation of vascular endothelial growth factor (VEGF) gene transcription by estrogen receptors alpha and beta. Proc Natl Acad Sci USA 97: 10972-10977, 2000 